\title{
Open-access paediatric echocardiography: changing role and referral patterns to a consultant-led service in a tertiary referral centre
}

\author{
A T Tybulewicz, M L Rigby, A N Redington
}

\begin{abstract}
Objectives-To evaluate the changing role of an open-access consultant-led paediatric echocardiography service for the detection and assessment of cardiac disease in children.

Design-A retrospective analysis comparing two patient groups undergoing echocardiography over two corresponding six month periods in 1989 and 1994.

Setting-A tertiary referral centre.

Main outcome measures-Patient demographics, indication for echocardiogram, source of referral and findings on scan in new referrals, subsequent follow up arrangements.

Results-The total workload increased by $51 \%$ over 5 years but in 1994 fewer neonates were scanned. Patients were referred by hospital paediatricians, community paediatricians, and general practitioners and the number of "new referrals" as a percentage of the total number of patients scanned remained constant. The number of children referred with asymptomatic murmurs who had "normal" echocardiograms increased. Fewer patients were referred directly for surgery in 1994, but the number of children referred for interventional catheterisation rose.

Conclusions-Open-access echocardiography has an expanding role in the tertiary referral centre despite increasing availability of echocardiography facilities in local hospitals and increased demand in local outreach clinics with paediatricians. Asymptomatic murmurs continue to be the single most common reason for referral of "new patients" and many scans are used to confirm the clinical suspicion of a "normal" heart. The appropriateness of using echocardiography as a screening procedure must be questioned where it would be more logical to refer only the children who present diagnostic difficulty. None the less these data confirm the impression of increasing demands on the paediatric cardiologist, and thus may be useful in planning consultant services within the specialty.
\end{abstract}

(Heart 1996;75:632-634)
Keywords: open access echocardiography; paediatrics; congenital heart disease

Cross sectional echocardiography has rapidly become established as the primary diagnostic technique for the investigation of children with heart disease. It is now routinely performed in the tertiary centre, and facilities and expertise often exist in the referral centre. Indeed, it is now commonplace for echocardiography to be available in the outreach clinic (a specialist clinic with local paediatrician and cardiologist). It is a general impression that these outreach clinics are becoming required more often and that more children are seen per clinic. Thus the need for a referral service in the tertiary centre should perhaps be reduced. In our hospital a weekly, open access echocardiography service has been available to general practitioners, community physicians, and district paediatricians for about 10 years. In this study we examine the workload of this service and the patterns of referral over a recent five year period.

\section{Methods}

The open access consultant-led echocardiography clinic is held weekly and takes referrals from all services, and doctors of all grades. There is no medical screening in the tertiary centre, each referral being booked into the next available clinic. A prerequisite is that an echocardiogram is requested by the referring doctor; all other referred patients attend the routine outpatient clinic (where echocardiography may or may not be performed). Two consultant paediatric cardiologists have been responsible for all scans performed, and the referral population has not changed during the study period.

Data were collected from the outpatient records of all children who attended the clinic during two six month periods (January-June 1989 and 1994). The latter time frame was chosen because this was the last six month period before the potential referral population increased (in the form of additional outreach clinics from new referring hospitals). The records were reviewed and the age of each patient and the recorded indication for echocardiogram was noted. In the case of "new referrals", the source of referral was recorded (hospital paediatrician, community
Correspondence to:

Dr A N Redington, Royal Brompton Hospital, Sydney

Accepted for publication

22 January 1996 
paediatrician, or general practitioner) and the echocardiogram result categorised as normal or abnormal. (A normal scan being one in which no underlying structural or functional abnormality was detected). In the children in whom symptoms had been noted which could be attributable to cardiac disease, only the predominant symptom was recorded. Only patients referred for routine studies were included (urgent cases generally were reviewed on the cardiac ward or paediatric intensive care unit); although clearly some patients were discovered to have severe abnormalities requiring urgent action. In the group of children who had previous scans, we noted whether the child was being followed up for a cardiac defect or after operation.

In all cases the plan for follow up was noted. This included any plan to admit for routine/ emergency surgery, cardiac catheterisation, and other interventional procedures or investigations.

\section{STATISTICAL ANALYSIS}

We used the Chi squared test to compare results in the two groups. The null hypothesis was rejected when $\mathrm{P}<0.05$.

\section{Results}

In all, 563 patients were seen in the clinic: 224 patients in 1989 and 339 in 1994. This reflects a $51 \%$ increase in workload over five years. Because 23 clinics were held during 1989 and 24 clinics in 1994 more patients were seen in the same allocated clinic time in $1994(9 \cdot 7$ patients in $1989 v 14.1$ patients in 1994).

\section{AGE AT REFERRAL}

Overall the age distribution of the patients being scanned in the two periods was similar except that there was a marked reduction in the number of patients being scanned in their first week of life in $1994(7.5 \% v 0.6 \%$ of total, $P<0.05)$.

INDICATION AND REFERRAL PATTERNS

Follow up of known patients accounted for $35 \%(63 / 178)$ and $38 \%(130 / 339)$ of the workload in 1989 and 1994 respectively and new patient referrals remained constant $(65 \%$ of total cases in 1989 and $62 \%$ in 1994) There was a tendency for more referrals of patients with asymptomatic murmur $(34.4 \% v 50.2 \%$, $P=0.07)$ and a reduction in those with symptoms attributable to heart disease $(16.3 v$ $6 \cdot 3 \%, P=0.001$ ).

The patients referred to this open access paediatric echocardiography clinic (table) originate largely from the district general hospitals with whom we hold outreach clinics

Outcome of scan (normal/abnormal) by source of referral in new patients (values are numbers (percentages))

\begin{tabular}{lllll}
\hline Source of referral & $1989(n=114)$ & Abnormal & $1994(n=203)$ & Normal \\
\hline Normal & Abnormal \\
General paediatrician & $38(33 \cdot 0)$ & $71(61 \cdot 7)$ & $103(50 \cdot 7)$ & $78(38 \cdot 4)$ \\
Community & $4(3 \cdot 5)$ & $1(0 \cdot 9)$ & $11(5 \cdot 4)$ & $6(3 \cdot 0)$ \\
\hline
\end{tabular}

(average $92 \%$ for 1989 and 1994) with a few from community paediatricians and general practitioners in the catchment area. The proportion of normal scans in this group of new referrals (irrespective of source of referral) was $37 \%(43 / 115)$ and $57 \%(120 / 209)$ in 1989 and 1994 respectively, $(P<0.05)$. It was not possible in the case of referrals from other hospitals to determine at which medical grade the referral had originated, but the number of scans that were normal in this group increased from $33 \cdot 0 \%$ to $50 \cdot 7 \%$.

\section{ECHOCARDIOGRAPHIC FINDINGS IN NEW} PATIENTS

The detection of abnormalities fell from $75 \%$ in 1989 to $51 \%$ in $1994(\mathrm{P}<0.01)$. In 1994 children presenting with a murmur and symptoms which could be attributable to cardiac disease represented a smaller percentage of referrals but the yield of abnormal scans from this group as a whole was similar to the asymptomatic group and had not changed over five years $(P=N S)$. Interestingly, the group with tachypnoea and a murmur (the most common symptom noted) had abnormalities in $92.3 \%$ in 1989 and $88.9 \%$ in 1994 ; this seems to be a good discriminator of disease. Cyanotic episodes showed an inconsistent yield of abnormal results and other symptoms were reported in too few cases to comment. The number of children being assessed for cardiac involvement in the presence of clinical and haematological features of Kawasaki disease has increased, accounting for $1 / 115(0.9 \%)$ of new referrals in 1989 and $10 / 203(4.9 \%$ $P<0.05)$ in 1994.

FOLLOW UP

Subsequent outpatient clinic appointments were made for $46.6 \%$ children in 1989 and $42.5 \%$ in 1994 . The clinic visits were to the Royal Brompton Hospital in the paediatric outpatient department, to an outreach cardiac clinic held at the child's local district-general hospital, or to a general paediatric outpatient clinic held at the local district general hospital. In $198918 \%$ of children were discharged from the clinic and in $199434.8 \%(P<0.001)$, reflecting the increase in normal scans. Children for whom follow up was planned in the echocardiogram clinic were largely those in whom an echocardiogram was an essential part of the examination-for example, Kawasaki disease, cardiomyopathy, post-operative assessment of coil-occluded arterial duct. The number of children for whom an admission for cardiac catheterisation was arranged fell slightly $(P=N S)$ but referrals incorporating an interventional procedure accounted for 8/21 in 1989 and $21 / 26$ in $1994(38 v 81 \%, \mathrm{P}<0.01)$. The reduction in admissions for surgery from $17 \cdot 4 \%$ in 1989 to $5.0 \%$ in $1994(P<0.001)$ may reflect the increase in the proportion of interventional versus assessment catheterisations.

\section{Discussion}

An open access to a consultant-led paediatric echocardiography service (with a short waiting 
time for an appointment) remains popular among referring physicians despite an increasingly widespread provision of echocardiography services in their local hospitals. This study shows that the number of such referrals to the tertiary referral centre increased. The fact that this service is provided by a paediatric cardiologist who is able to examine the child, perform and interpret the echocardiogram, and discuss its findings with parents in a single consultation, in a centre where operative and other interventional procedures can be performed if necessary, may influence the decision for such a referral. Other factors may include increasing parent and physician awareness of ultrasound scanning, as well as the reassurance factor of the scan result. No matter what the underlying reasons, it is clear from our data that this service is increasingly demanded, even though most patients referred are asymptomatic.

The practice of referral of children with an asymptomatic murmur for echocardiography is controversial ${ }^{1-3}$ and it has been suggested that the number of children being scanned in this group could be reduced if primary care physicians referred children first to a local paediatric cardiology outpatient clinic. ${ }^{2}$ Clinical examination by a paediatric cardiologist is a sensitive and specific discriminator between pathological and innocent murmurs and only a small number of scans would subsequently be requested. However, although not quantified in this study, the number of such outreach clinics performed, and the number of patients seen in each clinic has also risen. Thus the patients referred to the tertiary centre are the tip of an iceberg, and it seems that both tip and iceberg are increasing in size. Not surprisingly, the number of patients discharged from follow up has also increased. Whether a prompt referral for a definitive diagnostic procedure ultimately reduces the total burden on paediatric services is difficult to quantify. It is likely, however, that prompt discharge from medical follow up prevents unnecessary repeated clinical reviews and re-referral by general practitioners, community paediatricians, and school doctors. So while the total paediatric cardiology workload increases, the service to the patient and purchaser has probably improved also.

In those patients with abnormalities the subsequent pattern of management has also changed. The percentage of patients referred for interventional catheterisation procedures has increased significantly. At the same time the number of referrals for surgery has fallen, presumably reflecting the impact that interventional procedures have had on the need for surgery, particularly in this outpatient population with less complex structural abnormalities.

In conclusion, over five years there was an increase in the need for both outpatient echocardiography and inpatient interventional catheter procedures, which increased the cardiological workload in tertiary referral centres. The reasons are multifactorial and these data may be helpful in future planning of consultant manpower.

We thank Mr Manjit Josen for technical support and Ms Marjan Dezyanian for her secretarial help.

$1 \mathrm{Xu} \mathrm{M}$, Mhaffie DJ. Non-specific systolic murmurs; an audit of clinical value of echocardiography. $N Z \mathrm{Med} \mathcal{F}$ 1993;106:54-6.

2 Danford DA, Nasir A, Gumbiner C. Cost assessment of the evaluation of heart murmurs in children. Pediatrics 1993;2:365-8.

3 Sutherland GR. Has echo/Doppler influenced the practice of paediatric cardiology? Int $\mathcal{f}$ Cardiac Imaging 1993; 